Reprinted from Journal of Appined Physics, Vol. 32, No. 1, 75-82, January, 1961

Copyright 1961 by the American Institute of Physics

Printed in U. S. A

\title{
Reflection and Transmission of Electromagnetic Waves at Electron Density Gradients*
}

\author{
Frank A. Albini and Robert G. Jahn \\ Daniel and Florence Guggenheim Jet Propulsion Center, California Institute of Technology, Pasadena, California
}

(Received October 10, 1960)

\begin{abstract}
Solutions are obtained for the propagation of plane electromagnetic waves parallel to a gradient of free electron density, in the form of complex Airy functions. Reflection and transmission coefficients are derived for normal incidence on a linear "ramp" of electron clensity connecting a uniform dielectric gas with a uniform ionized gas, as functions of ramp length and propagation exponent of the latter. Machine evaluations of typical cases of physical interest are displayed and discussed. Similar study is made of two-stage ramps of variable proportions, intended as second approximations to smooth profile transition zones. In each case, the reflection and transmission coefficients are found to
\end{abstract}

\section{INTRODUCTION}

I many situations of current interest in which electromagnetic waves are caused to propagate into bodies of ionized gas, the transition from dielectric to dissipative medium is not abrupt, but extends over distances comparable with the wavelength. Typical examples arise in the microwave diagnostics of ionized gas flows in shock tubes, plasma tunnels, and fusion machines, where the probing wave train enters and leaves the medium of interest through thermal boundary layers embodying free electron density gradients. Similar charge density gradients are encountered by

* Work supported in part by the U. S. Air Force Office of Scientific Research. depend strongly on ramp width over a range of several tenths of a wavelength, then to oscillate mildly toward the asymptotic values predicted from a WKB-type approximation. The results ars less sensitive to the detailed shape of the electron density profile. Propagation through a finite slab of ionized gas bounded on each side by such linear transition zones is formulated and evaluated for typical cases. Asymptotic approximations for the linear ramp problem are found to be inadequate to cover the entire range of interest. The neglect of variation in collision frequency through the transition is discussed and justified for a broad class of equilibrium profiles.

radar signals intercepting ionized wakes, or long radio waves entering the ionosphere. In each case, if the gradient is mild and extends over many wavelengths, a WKB-type approximation will adequately represent the propagation in the nonuniform region. If the width of the transition zone is strictly comparable with one wavelength, however, an internal interference mechanism becomes important and neither the WKB approach nor a discrete interface treatment is applicable.

The purpose of this work is to derive and compute the reflection and transmission coefficients for exemplary transition zones between dielectric and dissipative gases. The analysis will be confined to harmonic plane electromagnetic waves propagating parallel to the gradients in free electron density, i.e., normal incidence. 
It will be assumed that the free electrons are the only particles interacting significantly with the driving field, that the magnetic forces on them are negligible compared with the electric forces, and that they collide predominantly with neutral particles. Although the problem is formulated in terms of ionized gas parameters, the treatment would be appropriate for any medium which could be characterized by a complex propagation exponent varying in the same manner. In fact, the equations and results are formally applicable to a variety of problems in other areas of physical interest.

\section{WAVE EQUATIONS}

Within the restrictions outlined in Sec. I, the highfrequency electrical properties of an ionized gas may be embodied in a linear conductivity function dependent on the frequency of the field, the free electron density, and some collisional parameter characterizing the transfer of momentum from the electrons to the bulk of the gas. The simplest formulation, similar to that proposed by Lorentz for bound electrons, ${ }^{1}$ stems from an heuristic equation for the drift velocity of an "ensemble-average" electron in an alternating field:

$$
m d \mathbf{v} / d t+m \nu_{c} \mathbf{v}=-e \mathbf{E} e^{i \omega t} .
$$

The quantity $\nu_{c}$ in the damping term is an effective collision frequency of the "average" electron with neutrals, and can be evaluated rigorously only by insertion of the appropriate velocity dependent collision cross sections in the Boltzmann equation. ${ }^{2,3}$ It is, however, a convenient parameter for characterizing the medium, and can frequently be estimated with sufficient accuracy. Integration of Eq. (1) leads to a current density which, upon comparison with the

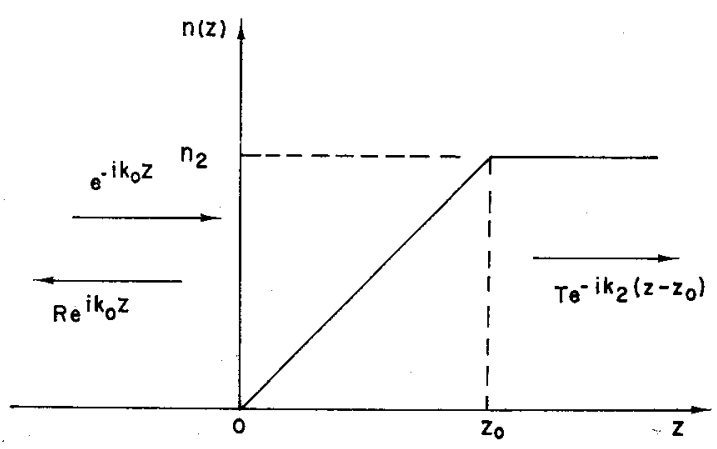

FIG. 1. The "linear ramp" geometry. Wave of unit amplitude incident from left on constant gradient of electron density connecting uniform media.

${ }^{1}$ H. A. Lorentz, The Theory of Electrons (B. G. Teubner, Leipzig, Germany, 1909), p. 306 ff.

${ }^{2}$ H. Margenau, Phys. Rev. 69, 508 (1946); 109, 6 (1958).

${ }^{3} \mathrm{~S}$. Chapman and T. G. Cowling, The Mathematical Theory of Non-Liniform Gases (Cambridge University Press, New York, 1952), (hap. 18. driving field, yields a complex conductivity

$$
\sigma\left(\omega, n, \nu_{c}\right)=\left[\frac{n e^{2}}{m\left(\omega^{2}+\nu_{c}^{2}\right)}\right]\left(\nu_{c}-i \omega\right),
$$

indicative of the in-phase and out-of-phase components of the electron current, both of which are linear in the electron density, $n$.

If $\sigma$ is uniform throughout the medium, the usual manipulation of Maxwell's equations ${ }^{4}$ yields a vector wave equation

$$
\nabla^{2} \mathbf{E}+k_{0}^{2}[1-i(\sigma / \epsilon \omega)] \mathbf{E}=0
$$

which permits plane transverse solutions of the form

$$
E=E_{0} e^{i(\omega t-k z)}
$$

where the complex propagation exponent $k$, which determines both wavelength and attenuation, is simply related to $\sigma$ :

$$
k=k_{0}[1-i(\sigma / \epsilon \omega)]^{\frac{1}{2}} .
$$

In this formulation, the values of $k_{0}\left(=2 \pi / \lambda_{0}=\omega / c_{0}\right)$, the permittivity $\epsilon$, and magnetic permeability $\mu$ are those of a nonconducting gas, i.e., essentially the free space values.

If gradients in free electron density, and hence in $\sigma$, exist, Maxwell's equations must be re-examined to determine permitted modes of propagation. For harmonic waves, the vector wave equation for $\mathbf{E}$ takes the form

$$
\nabla^{2} \mathbf{E}+k_{0}{ }^{2}\left[1-i\left(\frac{\sigma}{\epsilon \omega}\right)\right] \mathbf{E}+\nabla\left[\frac{\nabla \sigma \cdot \mathbf{E}}{\sigma+i \epsilon \omega}\right]=0
$$

which, for the special case of our interest, $\nabla \sigma \cdot \mathbf{E}=0$, returns to the form of $\mathrm{Eq}$. (3), with $\sigma$ now a function of z. More specifically, we restrict ourselves at first to the "linear ramp" geometry shown in Fig. 1, in which the

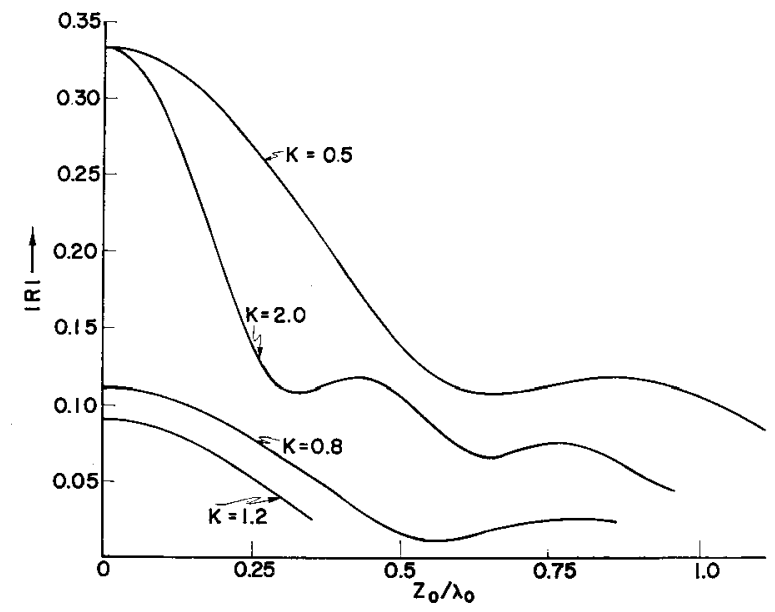

FIg. 2. Reflected amplitude vs ramp width for representative cases of pure real $K$.

${ }^{4} \mathrm{~J}$. A. Stratton, Electromagnetic Theory (McGraw-Hill Book Company, Inc., New York, 1941), n. 268 ff. 
free electron density rises linearly from zero at $z=0$ to a value $n_{2}$ at $z=z_{0}$ and thereafter is constant, while the collision frequency $\nu_{c}$ is assumed constant throughout (see Sec. VII). The wave equation within this ramp may be written

$$
\frac{d^{2} E}{d z^{2}}+k_{0}^{2}\left\{1-\left[1-K^{2}\right] \frac{z}{z_{0}}\right\} E=0
$$

where

$$
K=k_{2} / k_{0}=[1-i(\sigma / \epsilon \omega)]^{\frac{1}{2}}
$$

and can be reduced by the change of variable

$$
V=-\left[\frac{k_{0} z_{0}}{1-K^{2}}\right]^{\frac{2}{3}}\left\{1-\left[1-K^{2}\right] \stackrel{z}{z_{0}}\right\}
$$

to the Airy form

$$
d^{2} E / d V^{2}=V E
$$

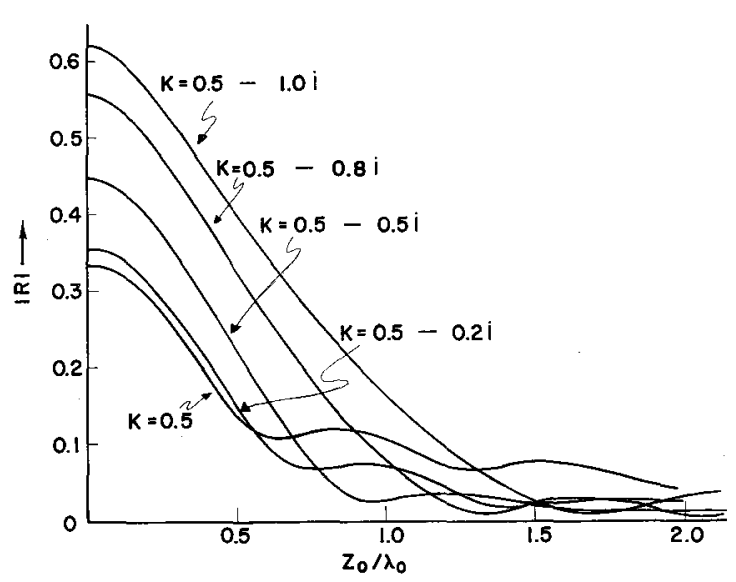

Fic. 3. Reflected amplitude vs ramp width for $K_{r}=0.5$ and various amounts of attenuation, $K_{i}$.

Solutions are available as tabulated Airy functions ${ }^{5}$

$$
E=A[a i(V)]+B[B i(V)]
$$

which, like the Bessel functions, ${ }^{6}$ are quasi-periodic, slowly changing in wavelength and amplitude-a reasonable behavior for a wave in an inhomogeneous medium.

\section{COEFFICIENTS OF TRANSMISSION AND REFLECTION}

A wave of unit amplitude $e^{-i k_{0} z}$ is assumed incident on the ramp from the left. A reflected wave of form $R e^{i k_{0} z}$, and a transmitted wave $T e^{-i k_{2}\left(z-z_{0}\right)}$, where $R$ and $T$ are in general complex quantities indicative of

\footnotetext{
${ }^{5}$ P. M. Woodward and A. M. Woodward, TRE Rept. T 1800 , Telegraphy Research Establishment, Malvern, England.

${ }^{6}$ The solutions could be written equally well in terms of Bessel functions of order $\frac{1}{3}$ and $-\frac{1}{3}$, or as J. Wallot [Ann. Physik 60, 734 (1919)] has done for a pure dielectric problem (or see Stratton, p. 580 of work cited in footnote 4), in terms of Hankel Functions. The Airy formulation was chosen because appropriate tables for complex arguments were more readily available.
}

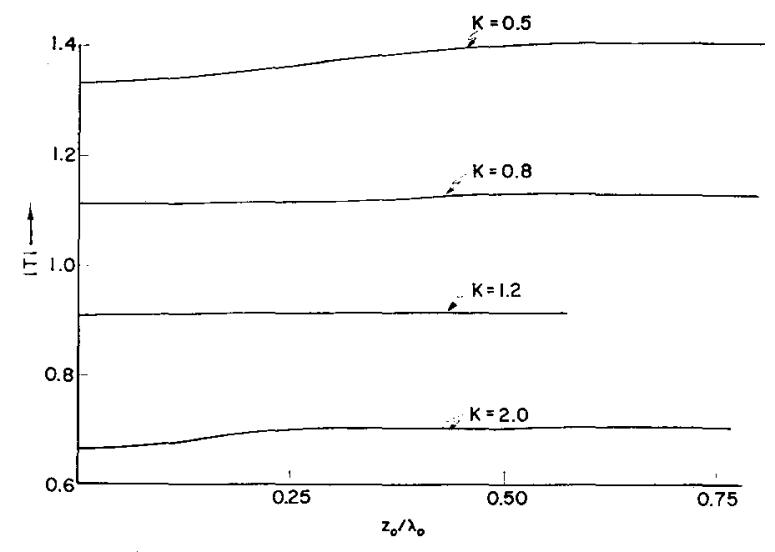

Fig. 4. Transmitted amplitude vs ramp width for representative cases of pure real $K$.

both amplitude and relative phase, are assumed to emerge from the ramp zone. The appropriate boundary conditions at the ramp edges are continuity of the electric and magnetic field vectors, which in the absence of any discontinuity in $k(z)$ are equivalent to continuity of $E$ and its first $z$ derivative. From the four resulting equations, $R$ and $T$ were evaluated numerically for representative cases on an IBM 709. The machine was programed to generate its own Airy functions in the complex plane from enough terms of the appropriate infinite series to guarantee less than 0.0001 error in each function. These were then combined in Argand form to return moduli and phases of $R$ and $T$. Calculations were carried out over the range of $k_{0} z_{0}$ from 0.2 to 15.0 in steps of 0.2 , for a network of values of $K=K_{r}-i K_{i}$ in the fourth quadrant.

Typical examples of the dependence of the reflected and transmitted amplitudes on ramp width, expressed in units of free space wave lengths $\lambda_{0}=2 \pi / k_{0}$, are shown in Figs. 2-5. In each case, the curves approach the proper discrete interface values at $\left(z_{0} / \lambda_{0}\right) \rightarrow 0$, and become asymptotic to the WKB solutions for large arguments. In the intermediate range there are'small

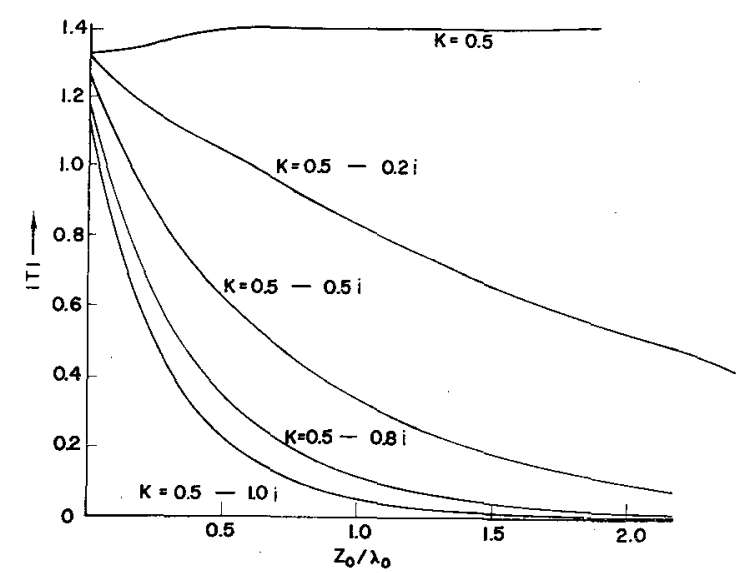

Frg. 5. Transmitted amplitude vs ramp width for $K_{r}=0.5$ and various amounts of attenuation, $K_{i}$. 


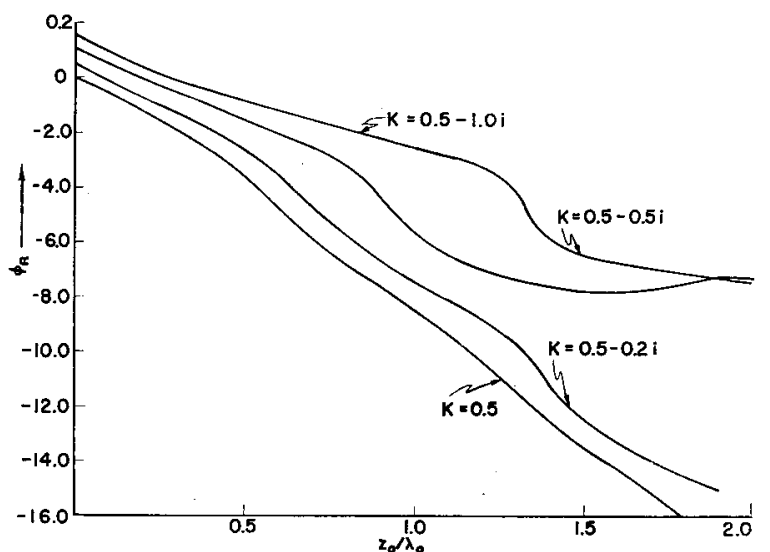

FIG. 6. Phase of reflected wave vs ramp width for $K_{r}=0.5$ and various amounts of attenuation, $K_{i}$.

periodic fluctuations-most pronounced for the cases of pure real $K$, subdued in the cases of large dampingwhich suggest interference between the two discontinuities in slope of $k(z)$, somewhat analogous to the more familiar interactions between discrete interfaces. In all cases, the reflected amplitude is found to decrease steeply over the first few tenths of a wavelength of ramp thickness abscissa, emphasizing the importance of including this parameter in the analyses of relevant physical situations. This effect is felt to arise from a smaller scale interference process distributed over the belt of inhomogeneity; i.e., the protraction of an interface into a more gradual transition spoils the coherence of the oscillators which give rise to the reflection field, and the aggregate amplitude is less.

Samples of the dependence of the phases of the reflected and transmitted waves on ramp width are shown in Figs. 6 and 7. The reflected phase, like the amplitude, undergoes periodic undulations as the ramp width is increased, eventually approaching a smooth asymptotic curve determined by the specific values of the real and imaginary parts of $K$. The transmitted phase, however, is found to be almost linearly de-

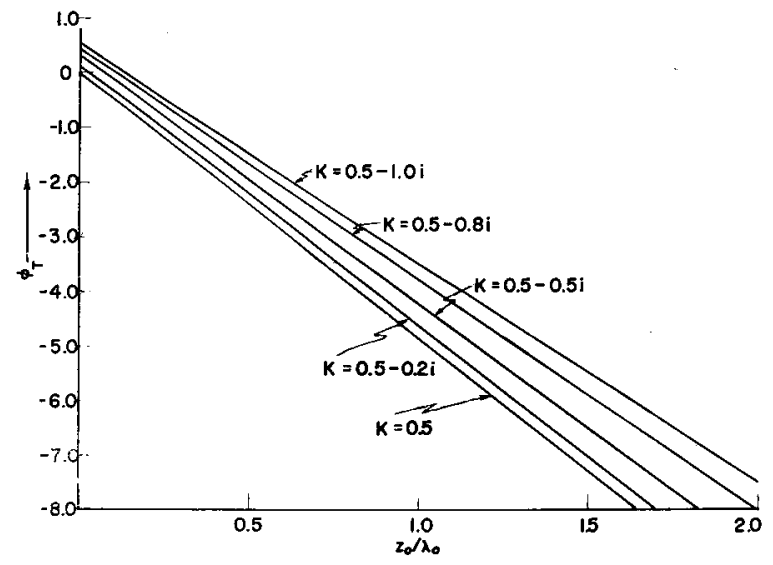

FIG. 7. Phase of transmitted wave vs ramp width for $K_{r}=0.5$ and various amounts of attenuation, $K_{i}$. pendent on ramp width, with a slope comparatively insensitive to the imaginary part of $K$. The values of this phase can be estimated surprisingly well by a simple average of the propagation exponent over the ramp:

$$
\begin{aligned}
& \phi_{T}=\phi_{D}-\operatorname{Re}\left[\int_{0}^{z_{0}} k(z) d z\right] \\
&=\phi_{D}-\operatorname{Re} e\left[\frac{2 k_{0} z_{0}}{3}\left(1+\frac{K^{2}}{1+K}\right)\right],
\end{aligned}
$$

where $\phi_{D}$ is the phase shift through a discrete interface between the same media, $=\tan ^{-1}\left[K_{i} /\left(1+K_{r}\right)\right]$. As such, the transmitted signal has the same phase as it would had it emerged from such a discrete interface, located at a position within the ramp, $z_{e}$, determined by $K$ :

$z_{e}=\frac{\phi_{D}-\phi_{T}-K_{r} z_{0}}{1-K_{r}}=z_{0}\left\{\frac{\left\{\frac{2}{3} \operatorname{Re}\left(1+\frac{K^{2}}{1+K}\right)-K_{r}\right]}{1-K_{r}}\right\}$

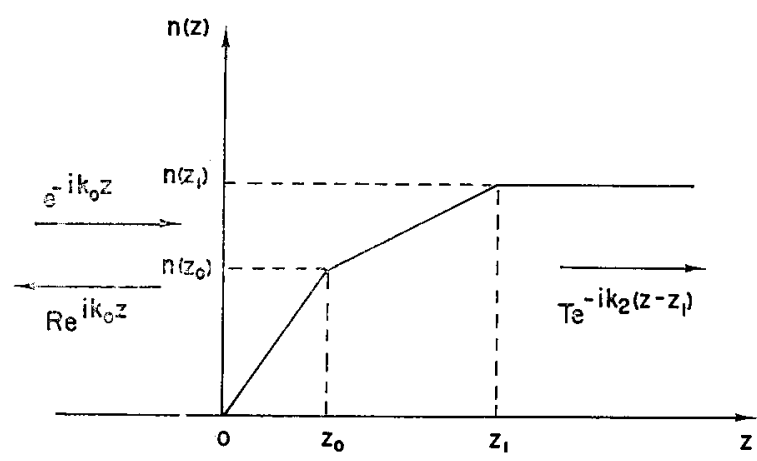

FIG. 8. The "kinked ramp" geometry. Wave of unit amplitude incident from left on piecewise linear variation of electron density connecting two uniform media.

For real $K$, this equivalent interface is confined between the extremes, $z_{0} / 3$, for $K_{r} \rightarrow \infty$, and $2 z_{0} / 3$, for $K_{r} \rightarrow 0$.

\section{KINKED RAMP}

The strong, irregular dependence of $R$ and $T$ on ramp width found for this first crude representation of a transition zone justifies examination of their sensitivity to finer details of the electron density profile. Rather than attempting solution for any curved profile of $K(z),{ }^{7}$ a "kinked ramp" geometry was studied (Fig. 8), which by variation of the $z$ and $K$ coordinates of the kink could serve as a piecewise linear second approximation to a variety of smooth transitions. Wave equations similar to Eq. (7) in the two linear segments

\footnotetext{
${ }^{7}$ Wallot's treatment (see footnote 6) of the similar problem for more general profiles of permittivity, $\epsilon(z)$, is not transcribable to a variable conductivity, since the concomitant variation of $n(z)$ imposed by Eq. (2) would need be complex. P. Poincelot [Compt. rend. 244, 2031, 2298, 3045 (1957)] considers more general profiles of $\sigma(z)$ that extend to infinity, and hence lack the second boundary essential to the problem under study here.
} 


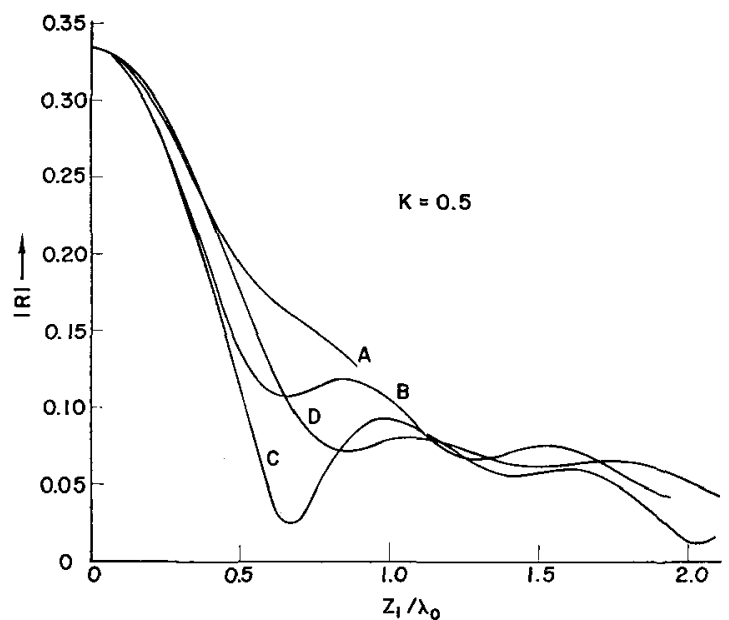

FIG. 9. Reflected amplitude vs total width of "kinked ramp" of $K=0.5$ for various kink coordinates (see Fig. 8) : (A) $n\left(z_{0}\right) / n\left(z_{1}\right)$ $=0.2 ; z_{0} / z_{1}=0.4$. (B) $n\left(z_{0}\right) / n\left(z_{1}\right)=0.4 ; z_{0} / z_{1}=0.4$. (C) $n\left(z_{0}\right) / n\left(z_{1}\right)$ $=0.6 ; z_{0} / z_{1}=0.4$. (D) $n\left(z_{0}\right) / n\left(z_{1}\right)=0.8 ; z_{0} z_{1}=0.4$.

were matched across the three boundaries, and thence $R$ and $T$ were evaluated for all combinations of the kinked ramp parameters shown in Table I. Values of reflection and transmission moduli for representative cases are shown in Figs. 9-11. In general, the results are qualitatively similar to those for the linear ramp, and reproduce them exactly for those combinations of parameters which remove the kink, i.e., for equal gradients in the two parts of the ramp. Again we find the sharp decrease in $|R|$ near the origin, followed by periodic fluctuations toward the asymptotic values. In most cases, the values are relatively insensitive to the coordinates of the kink over the first few tenths of a wavelength of $z_{1}$, suggesting that the linear ramp theory is an adequate first approximation over this range for any moderate profile of electron density.

The phases of the reflected and transmitted waves also resemble those for the linear ramp. Prominent periodicities appear in the former, and the latter are almost linearly dependent on ramp thickness. The simple integration of $k(z)$ over the two portions of the ramp, as in Eq. (11), again gives accurate values for $\phi_{T}$ vs $k_{0} z_{1}$, indicating that similar technique may be valid for arbitrary smooth profiles of $n(z)$.

\section{TRAPEZOID GEOMETRY}

In many of the physical situations of interest, the extent of the body of ionized gas in the direction of propagation is short enough that the electromagnetic

TABLE I. Grid of "kinked ramp" calculations.

\begin{tabular}{|c|c|c|c|c|}
\hline$K 1_{r}$ & $-\dot{K}_{1}$ & $k_{0} z_{1}$ & $z_{0} / z_{1}$ & $n\left(z_{0}\right) / n\left(z_{1}\right)$ \\
\hline 0.5 & 0 & 0.2 to 15.0 & 0.2 & 0.2 \\
\hline 0.8 & 0.1 & $\{$ in steps of & 0.4 & 0.4 \\
\hline \multirow[t]{2}{*}{2.0} & 0.2 & 0.4 & 0.6 & 0.6 \\
\hline & 0.5 & & 0.8 & 0.8 \\
\hline
\end{tabular}

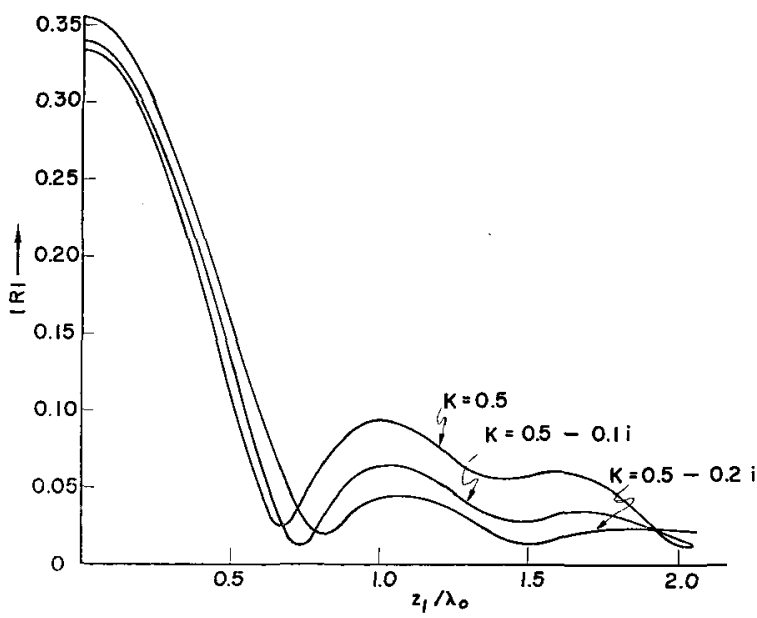

FIG. 10. Reflected amplitude vs total width of "kinked ramp" for various amounts of attenuation, $K_{i}$. Kink coordinates $n\left(z_{0}\right) / n\left(z_{1}\right)=0.6 ; z_{0} / z_{1}=0.4$ in each case.

wave can reach the back boundary before it is substantially attenuated. In such cases, the interaction with a second, inverse transition zone there also contributes to the reflected and transmitted signals. This contribution, of course, is not simply additive, but acts in interference with the corresponding interaction at the front transition zone, necessitating simultaneous solution of the entire wave pattern. The gross features can be anticipated from the known solutions for the limiting case of discrete interfaces, i.e., a plane slab of ionized gas. ${ }^{8}$ Graphs of such relations for typical values of $K$, shown in Figs. 12 and 13, display a strong interference pattern as a function of interface separation, on which is superimposed an attenuation characteristic of the interior medium.

The corresponding problem with the discrete interfaces replaced by linear ramp transitions (see Fig. 14) was formulated by matching plane wave solutions in

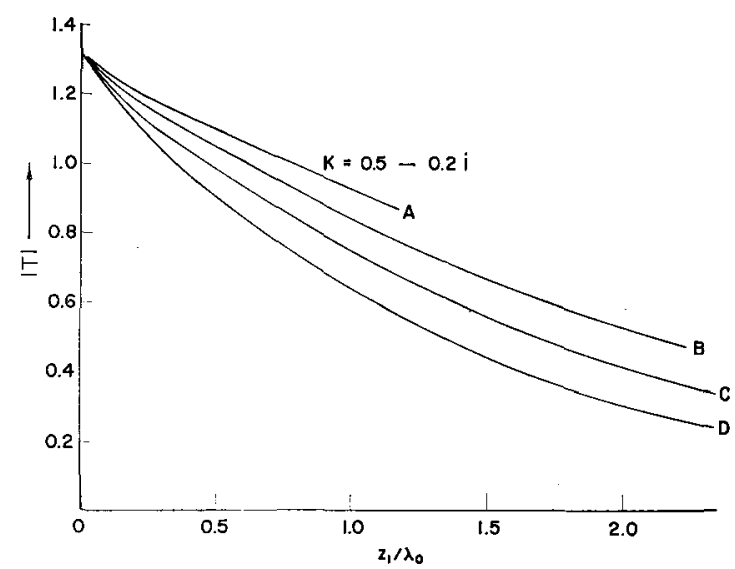

FIG. 11. Transmitted amplitude vs total width of "kinked ramp" of $K=0.5-0.2 i$ for various kink coordinates (see Fig. 8 ): (A) $n\left(z_{0}\right) / n\left(z_{1}\right)=0.2 ; z_{0} / z_{1}=0.4$. (B) $n\left(z_{0}\right) / n\left(z_{1}\right)=0.4 ; z_{0} / z_{1}=0.4$. (C) $n\left(z_{0}\right) / n\left(z_{1}\right)=0.6 ; z_{0} / z_{1}=0.4$. (D) $n\left(z_{0}\right) / n\left(z_{1}\right)=0.8 ; z_{0} / z_{1}=0.4$.

${ }^{8}$ J. A. Stratton, p. $511 \mathrm{ff}$. of work cited in footnote 4 . 


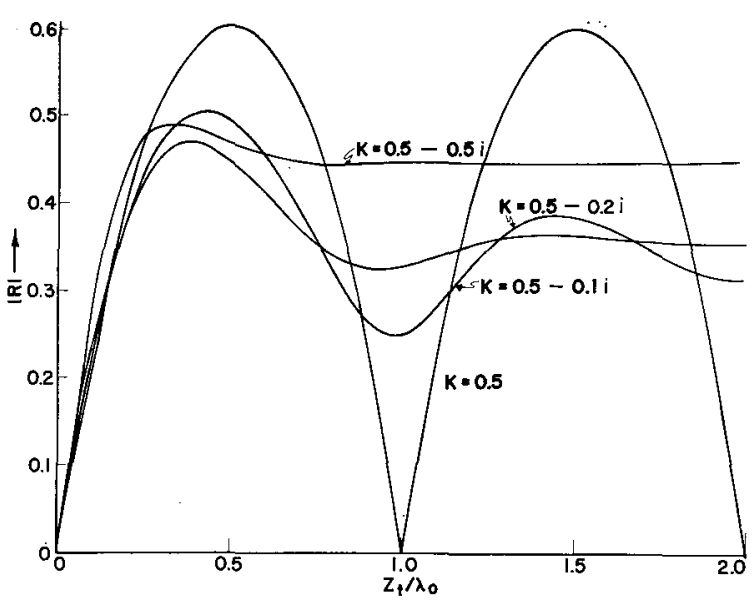

FIG. 12. Reflected amplitude vs slab width for $K_{r}=0.5$ and various amounts of attenuation, $K_{i}$.

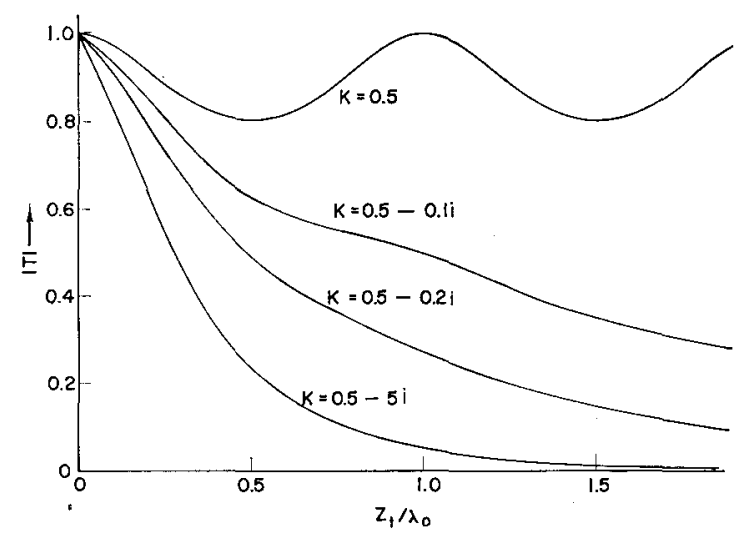

Fig. 13. Transmitted amplitude vs slab width for $K_{r}=0.5$ and various amounts of attenuation, $K_{i}$.

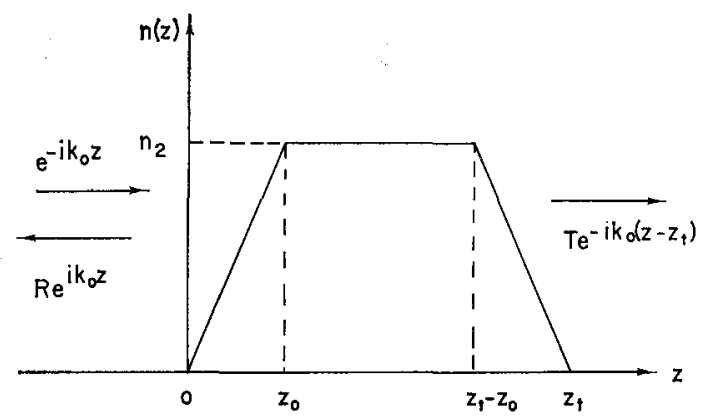

FIG. 14. "Trapezoid" geometry; uniform slab of ionized gas bounded by symmetric linear ramps of electron density.

the uniform media with the appropriate Airy functions in the ramps. Typical computed values of $R$ and $T$ are shown in Figs. 15 and 16, for an undamped case, and in Figs. 17 and 18 for a slightly attenuating medium. In the former, the effects of protracting the interfaces into linear ramps are a shift in the positions of maxima and minima relative to the $k_{0} z_{t}$ axis while retaining the same spacing, and a reduction in both the maxima of reflection and the minima of transmission. In Figs. 17 and 18 , the introduction of an imaginary component

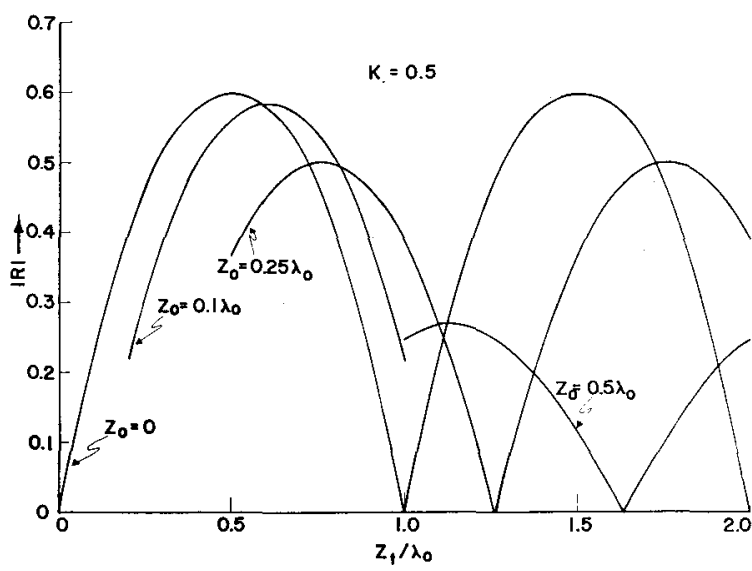

FIG. 15. Reflected amplitude vs total width of trapezoid for various widths of the boundary ramps; $K=0.5$.

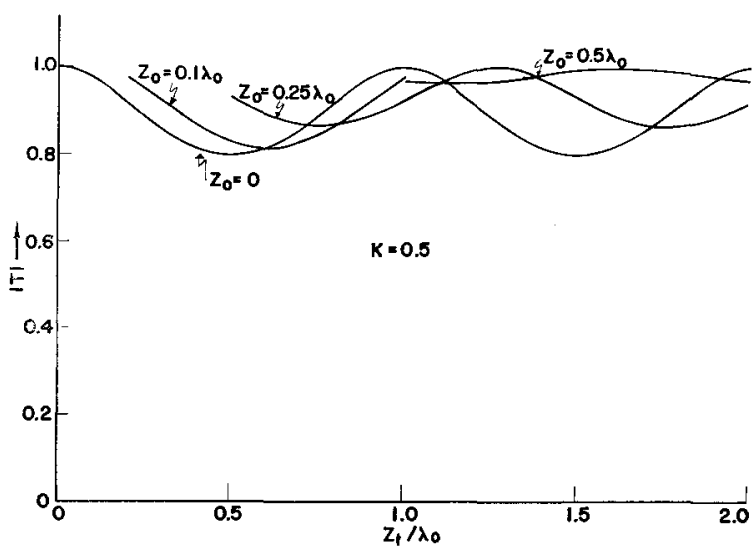

Frg. 16. Transmitted amplitude vs total width of trapezoid for various widths of the boundary ramps; $K=0.5$.

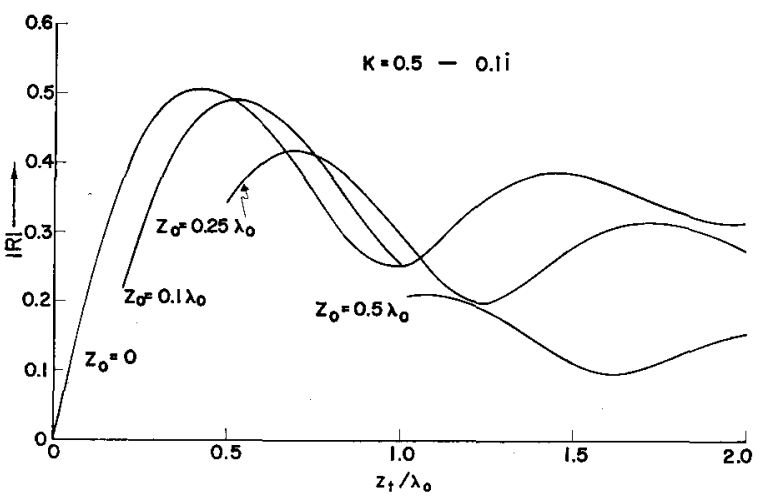

FIG. 17. Reflected amplitude vs total width of trapezoid for various widths of the boundary ramps; $K=0.5-0.1 i$.

of $K$ is seen to have the anticipated effect of attenuating the amplitude of both $R$ and $T$ as $k_{0} z_{t}$ is increased.

\section{ASYMPTOTIC SOLUTIONS FOR LINEAR RAMP}

Small argument expressions for $R$ and $T$ for the linear ramp may be generated by insertion of the appropriate expansions of the Airy functions into 
expressions for $R$ and $T$ derived from the boundary equations:

$$
\begin{aligned}
& R=R_{0}\left[\frac{1-(i / 2) k_{0} z_{0}(1-K)-\frac{1}{6}\left(k_{0} z_{0}\right)^{2}\left(1-K+K^{2}\right)+\cdots}{1+(i / 2) k_{0} z_{0}(1+K)-\frac{1}{6}\left(k_{0} z_{0}\right)^{2}\left(1+K+K^{2}\right)+\cdots}\right], \\
& T=T_{0}\left[1+(i / 2) k_{0} z_{0}(1+K)-\frac{1}{6}\left(k_{0} z_{0}\right)^{2}\left(1+K+K^{2}\right)\right]^{-1},
\end{aligned}
$$

where

and

$$
R_{0}=(1-K) /(1+K)
$$

$$
T_{0}=2 /(1+K)
$$

are the reflection and transmission coefficients for an abrupt interface. It is perhaps noteworthy that in the course of such algebra, which is essentially the same as that performed by the computer, all fractional powers of quantities involving $K$ drop out, thereby precluding any ambiguity in the choice of roots in the complex plane.

The convergence of Eqs. (13) is adversely affected by the complex coefficients and in general, the validity is restricted to $k_{0} z_{0}<1$. To carry enough additional terms to overlap the large argument approximations would be of comparable difficulty to the complete solution. The expressions are useful, however, in indicating the slopes of the amplitudes at the origin:

$$
\begin{aligned}
& \left.\frac{d|R|}{d\left(k_{0} z_{0}\right)}\right|_{0}=0, \\
& \left.\frac{d|T|}{d\left(k_{0} z_{0}\right)}\right|_{0}=-\left|T_{0}\right| \frac{K_{i}}{2} .
\end{aligned}
$$

Figures 19 and 20 compare the small argument approximations with the complete solutions for a typical case.

Large argument approximations were formed similarly by inserting the asymptotic representations of the Airy functions ${ }^{9}$ into the same expressions for $R$

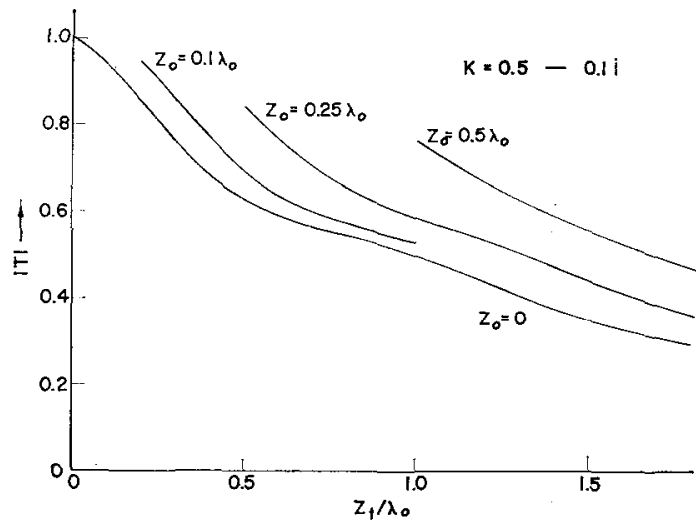

FIg. 18. Transmitted amplitude vs total width of trapezoid for various widths of the boundary ramps; $K=0.5-0.1 i$.

${ }^{9}$ A. Erdélyi, Asymptotic Expansions (Dover Publications, New York, 1956), p. 94 ff. and $T$, yielding, for $k_{0} z_{0} \gg\left|1-K^{2}\right|$,

$$
\begin{gathered}
R \sim \frac{-i\left(1-K^{2}\right)}{8 k_{0} z_{0}}\left\{1-K^{-3} \exp \left[-\frac{4 i}{3} k_{0} z_{0}\left(1+\frac{K^{2}}{1+K}\right)\right]\right\} \\
T \sim \frac{1}{K^{1}}\left[1+\frac{i\left(1-K^{3}\right)\left(1-K^{2}\right)}{8 K^{3} k_{0} z_{0}}\right] \\
\times \exp \left[-\frac{2 i}{3} k_{0} z_{0}\left(1+\frac{K^{2}}{1+K}\right)\right] .
\end{gathered}
$$

Restrictions on ranges of argument for validity of the chosen asymptotic forms translate into the requirement

$$
-\pi<\left[3 \arg K-\arg \left(1-K^{2}\right)\right]<0 .
$$

Other expansions are available for other ranges, but the bulk of the physically interesting cases fall within the above limits. Two exceptions of importance are the limiting case $K \rightarrow 1$, for which Eqs. (15) nevertheless give the correct values $R \rightarrow 0, T \rightarrow e^{-i k_{0} z_{0}}$ and the cases of pure imaginary $K$, for which $|R|=1$ for all. $k_{0} z_{0}$; i.e., regardless of ramp length, no power can be transmitted into the second medium. ${ }^{10}$

Evaluations of Eqs. (15) for $|R|$ and $|T|$ are also included in Figs. 19 and 20 in comparison with the exact solutions. The approximations are seen to be adequate for $\left(z_{0} / \lambda_{0}\right)>1$, but again, inclusion of more terms in the asymptotic expressions to extend the validity over the complete range of interest is sufficiently. cumbersome to justify the complete calculation.

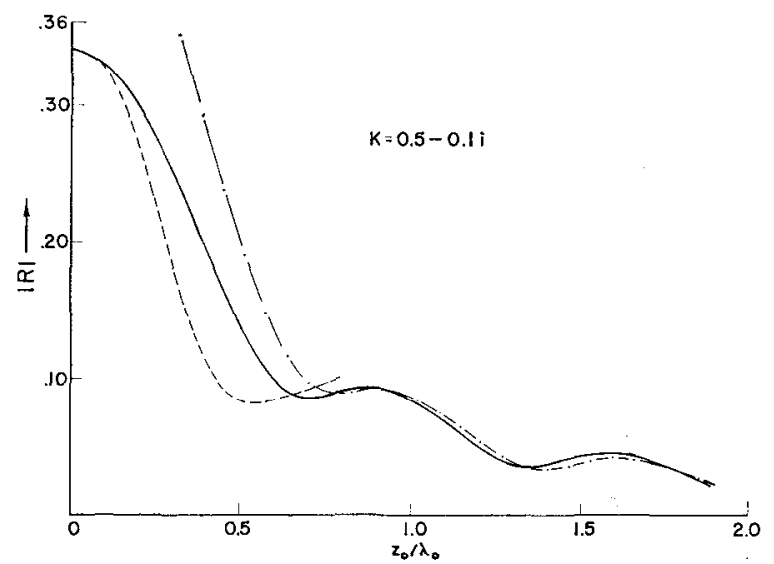

FIG. 19. Comparison of small and large argument approximations for $|R|$ (dashed curves), with the complete solution for the linear ramp; $K=0.5-0.1 i$.

${ }^{10}$ F. A. Albini and R. G. Jahn, Tech. Rept. No. 3, Guggenheim Jet Propulsion Center, California Institute of Technology, Pasadena, California, 1960. 


\section{VARIATION OF COLLISION FREQUENCY}

Of the several simplifications introduced in Sec. II, the most restrictive is the assumption of constant collision frequency through the transition zone. Because of the irregular dependence of the effective electronneutral collision cross section on temperature and type of gas molecule, the validity of a constant $\nu_{c}$ model must be appraised for specific physical situations. In a typical case, for example, the transition zone might involve a temperature gradient at constant pressure, wherein the gas is everywhere in local thermal equilibrium. Under these circumstances we may approximate:

$$
\nu_{c} \propto Q_{e}(T) T^{-\frac{1}{2}},
$$

where $Q_{\theta}(T)$ is the electron-neutral collision cross section, appropriately averaged over the electron velocity distribution. The electron density in a slightly ionized monatomic gas, on the other hand, is related almost exponentially to local temperature ${ }^{11}$ :

$$
n \propto T^{\frac{1}{4}} e^{-V_{I} / k T},
$$

where $V_{I}$ is the first ionization potential. From Eqs. (2) and (5), the explicit dependence of the propagation exponent $k$ on $n$ and $\nu_{c}$ may be written

$$
k=k_{0}\left[1+\frac{n e^{2}}{m \epsilon \omega}\left(\frac{1}{i \nu_{c}-\omega}\right)\right]^{\frac{1}{2}} .
$$

The comparative sensitivity of the problem to the $n$ and $\nu_{c}$ variations can then be estimated from the ratio of the partial gradients of $k$ at constant $\nu_{c}$ and $n$, respectively:

$$
\begin{aligned}
\frac{\left(\frac{\partial k}{\partial z}\right)_{v_{c}}}{\left(\frac{\partial k}{\partial z}\right)_{n}}=\frac{\left(\frac{\partial k}{\partial n}\right)_{\nu_{c}}\left(\frac{\partial n}{\partial T}\right)_{p} \frac{d T}{d z}}{\left(\frac{\partial k}{\partial \nu_{c}}\right)_{n}\left(\frac{\partial \nu_{c}}{\partial T}\right)_{p} \frac{d T}{d z}} \\
=\left(1+i \frac{\omega}{\nu_{c}}\right) \\
\left.\frac{\frac{V_{I}}{k T}+\frac{1}{2}}{1-2\left(\frac{d \ln Q_{e}}{d \ln T}\right)}\right) .
\end{aligned}
$$

Small ionization implies $V_{I} / k T \gg \frac{1}{2}$ throughout the transition. In view of the averaging over electron velocity implicit in $Q_{e}$, the logarithmic derivative is normally small, and as a rule of thumb

$$
\left(\frac{\partial k}{\partial z}\right)_{\nu_{c}} /\left(\frac{\partial k}{\partial z}\right)_{n}=\frac{V_{I}}{k T}\left(1+i \underset{\nu_{c}}{-\omega}\right)
$$

indicating that the collision frequency variation is indeed a secondary effect, particularly for the common situation of $\omega \gg \nu_{c}$.

11 S. C. Lin, E. L, Resler, and A. Kantrowitz, J. Appl. Phys. 26, 95 (1955).

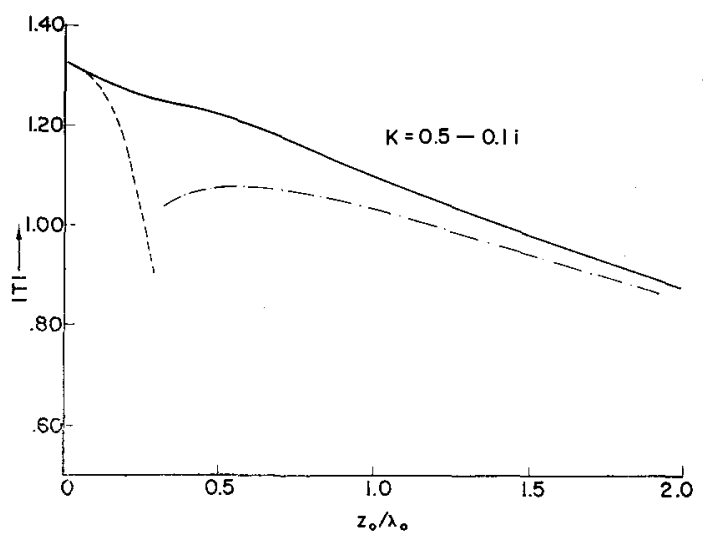

Fig. 20. Comparison of small and large argument approximations for $|T|$ (dashed curves), with the complete solution for the linear ramp; $K=0.5-0.1$.

The argument is less straightforward for other physical situations of interest, notably nonequilibrium transition zones. If necessary, it is possible to fabricate special functions $\eta(z)$ which embody variable $\nu_{c}(z)$, but care must be taken that the concomitant $n(z)$ imposed by the linear ramp formulation retains some physical significance.

\section{SUMMARY REMARKS}

The principal conclusions of this study-that the reflection and transmission of electromagnetic waves incident normally on a body of ionized gas depend strongly on the width of the transition zone at the boundary, less strongly on the detailed profile of the transition-have been illustrated by graphs of a few typical calculations. More details of the calculating procedures and more complete results for the linear ramp, kinked ramp, and trapezoid geometries are presented in a technical report ${ }^{10}$ from which quantitative interpolation to most physically interesting combinations of parameters can be made.

Systematic experiments suitable for comparison with the predictions of the foregoing models have not been found, although Haddenhorst has observed somewhat similar behavior for the reflection of guided waves from a structure of dielectric pyramids impregnated with carbon, ${ }^{12}$ and Greene and Hornig have demonstrated the insensitivity of $|R|$ to ramp profile for optical frequencies in a dielectric gas in the course of their shock thickness measurements. ${ }^{13}$ More directly applicable microwave experiments are currently in progress at this Laboratory.

\section{ACKNOWLEDGMENTS}

The authors acknowledge with thanks the help of Profs. J. Todd, W. D. Rannie, and F. E. Marble, and the staff of the Western Data Processing Center, U. C. L. A., on whose IBM 709 the calculations were performed.

${ }^{12}$ H. G. Haddenhorst, Z. angew Phys. 8, 264 (1956).

${ }^{13}$ E. Greene and D. Hornig, J. Chem. Phys. 21, 617 (1953). 